\title{
Molecular evolution in star-forming cores: From prestellar cores to protostellar cores
}

\author{
Yuri Aikawa ${ }^{1}$, Valentine Wakelam $^{2}$, Nami Sakai ${ }^{3}$, R. T. Garrod ${ }^{4}$, \\ E. Herbst ${ }^{5}$, and Satoshi Yamamoto ${ }^{3}$ \\ ${ }^{1}$ Department of Earth and Plantary Sciences, Kobe University, \\ Kobe 657-8501, Japan \\ email: aikawa@kobe-u.ac.jp \\ ${ }^{2}$ Université Bordeaux I \\ ${ }^{3}$ Department of Physics, University of Tokyo \\ ${ }^{4}$ Max-Planck-Institute für Radioastronomie \\ ${ }^{5}$ Departments of Physics, Chemistry, and Astronomy, The Ohio State University
}

\begin{abstract}
We investigate the molecular abundances in protostellar cores by solving the gasgrain chemical reaction network. As a physical model of the core, we adopt a result of onedimensional radiation-hydrodynamics calculation, which follows the contraction of an initially hydrostatic prestellar core to form a protostellar core. Temporal variation of molecular abundances is solved in multiple infalling shells, which enable us to investigate the spatial distribution of molecules in the evolving core. The shells pass through the warm region of $T \sim 20-100 \mathrm{~K}$ in several $10^{4} \mathrm{yr}$ and falls onto the central star in $\sim 100 \mathrm{yr}$ after they enter the region of $T>100 \mathrm{~K}$. We found that the complex organic species such as $\mathrm{HCOOCH}_{3}$ are formed mainly via grain-surface reactions at $T \sim 20-40 \mathrm{~K}$, and then sublimated to the gas phase when the shell temperature reaches their sublimation temperatures $(T \geqslant 100 \mathrm{~K})$. Carbon-chain species can be re-generated from sublimated $\mathrm{CH}_{4}$ via gas-phase and grain-surface reactions. $\mathrm{HCO}_{2}^{+}$, which is recently detected towards $\mathrm{L} 1527$, are abundant at $r=100-2,000 \mathrm{AU}$, and its column density reaches $\sim 10^{11} \mathrm{~cm}^{-2}$ in our model. If a core is isolated and irradiated directly by interstellar UV radiation, photo-dissociation of water ice produces $\mathrm{OH}$, which reacts with $\mathrm{CO}$ to form $\mathrm{CO}_{2}$ efficiently. Complex species then become less abundant compared with the case of embedded core in ambient clouds. Although a circumstellar (protoplanetary) disk is not included in our core model, we can expect similar chemical reactions (i.e., production of large organic species, carbon-chains and $\mathrm{HCO}_{2}^{+}$) to proceed in disk regions with $T \sim 20-100 \mathrm{~K}$.
\end{abstract}

Keywords. Stars: formation, ISM: molecules

\section{Introduction}

Chemical reactions in molecular clouds are classified to two categories: gas-phase reactions and grain-surface reactions. Figure 1 schematically summarizes the various chemical processes in molecular clouds. In the cold clouds of $T \sim 10 \mathrm{~K}$, chemical reactions are triggered by cosmic-ray ionization and proceed mainly via ion-molecule reactions in the gas-phase. While neutral-neutral reactions often have activation barrier, some neutralneutral reactions do not, and play an important role in forming molecules such as $\mathrm{N}_{2}$. When atoms and molecules in the gas-phase collide with grains in the cold molecular clouds, they are efficiently adsorbed onto grain surfaces. The adsorbed species migrate on the grain surface, and react with each other when they meet. Grain-surface reactions are considered to contribute significantly to the formation of large organic species, because grain surfaces are relatively enriched with heavy-element species, and because the association reactions $(\mathrm{AB}+\mathrm{C} \rightarrow \mathrm{ABC})$ are possible by depositing the excess energies on 
the grain surfaces. In the gas-phase reactions, on the other hand, products are mostly broken apart $(\mathrm{AB}+\mathrm{C} \rightarrow \mathrm{A}+\mathrm{BC})$ to discard the excess energies as kinetic energies of the products.

Efficiency of each process in Figure 1 varies significantly with physical conditions. In prestellar cores with high density $\left(n_{\mathrm{H}} \geqslant 10^{5} \mathrm{~cm}^{-3}\right)$ and low temperature $(T \sim 10 \mathrm{~K})$, the adsorption overwhelms the desorption, although the non-thermal desorption by impinging cosmic-rays, UV, and reaction heat of grain-surface reactions is not negligible. On the grain-surface, hydrogenation $(\mathrm{A}+\mathrm{H} \rightarrow \mathrm{AH})$ should be the dominat reaction; since hydrogen atoms have relatively low mass and low binding energy onto the grain surface, they can migrate efficiently even under low temperatures.

In protostellar cores, on the other hand, hydrogen atoms are thermally sublimated to the gas phase. Heavy-element species, with higher binding energies (onto grains) than hydrogen atoms, are still on the grain surface, migrating to react with each other. As the core temperature rises further, the heavy element species are also sublimated according to their volatility. The sublimates undergo gas-phase reactions, which have been considered to be the dominant formation path of the large organic species observed in hot cores in high-mass star forming regions (e.g., Millar \& Hatchell 1998). At the temperature of $200 \mathrm{~K}$ and density of $n_{\mathrm{H}} \sim 10^{6} \mathrm{~cm}^{-3}$, for example, large organic species are formed from sublimated $\mathrm{CH}_{3} \mathrm{OH}$ and $\mathrm{H}_{2} \mathrm{CO}$ by the gas-phase reactions in $\sim 10^{5}$ yrs. But it is not clear if a similar process works in low-mass protostellar cores, in which large organic species are detected in recent years (Ceccarelli et al., in this volume). In low-mass cores, the hot region $(T>100 \mathrm{~K})$ with sublimated $\mathrm{CH}_{3} \mathrm{OH}$ must be much smaller than in high-mass cores, and thus the infalling material would pass through such hot regions in a shorter timescale than $10^{5}$ yrs. Furthermore, the recent theoretical studies and laboratory experiments on chemical reactions suggest that the gas-phase formation of large organic species would not be as efficient as previously assumed (Horn et al. 2004, Geppert et al. 2006). Garrod \& Herbst (2006) showed grain-surface reaction at warm temperature $(\sim$ several $10 \mathrm{~K})$ can be an alternative or supplimental formation path of large organic species.

Molecular evolution in star-forming cores can be predicted quntitatively by solving the chemical reaction network, which includes the chemical processes mentioned above and are described mathematically as rate equations,

$$
\frac{d x(i)}{d t}=\sum_{j, k} \alpha_{j, k} x(j) x(k) n_{\mathrm{H}}+\sum_{j} \beta_{j} x(j) .
$$

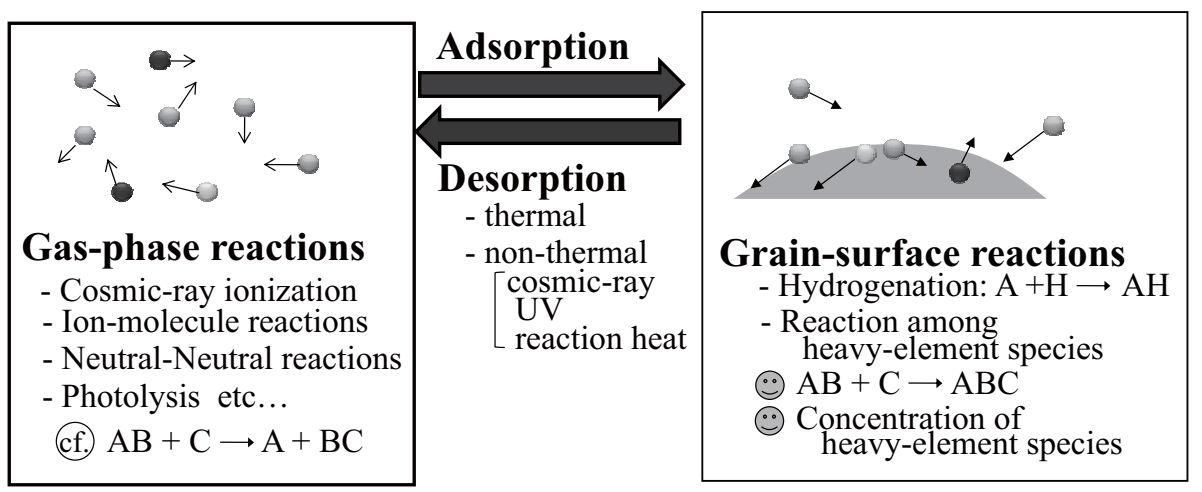

Figure 1. Chemical processes in molecular clouds. 

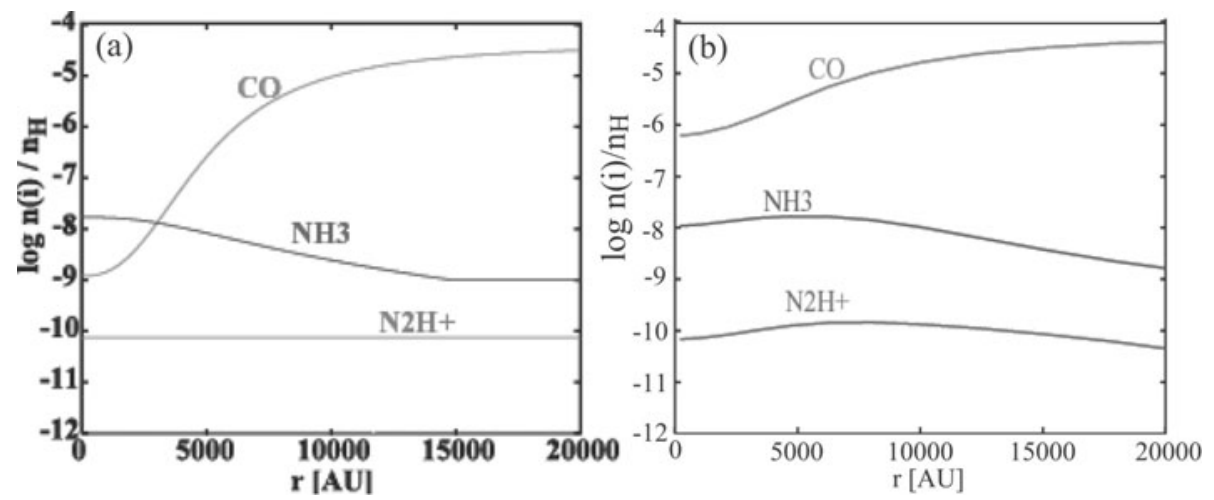

Figure 2. Radial distribution of molecular abundances in prestellar cores. Abundances derived from the observation of L1517B (Tafalla et al. 2002) (a) and our model (b).

The reaction rate coefficients, $\alpha$ and $\beta$, are in general a function of temperature, and $x(i)$ is the number density of species $i$ relative to hydrogen nuclei $\left(n_{\mathrm{H}}\right)$. The solution of Eq. (1) then depends on density $\left(n_{\mathrm{H}}\right)$ and temperature, which are often assumed to be constant or a simple function of time. Garrod \& Herbst (2006), for example, assumed that the density is constant and temperature rises as a square of the time $\left(T \propto t^{2}\right)$. While such simple assumptions on the physical conditions are convenient in investigating the dependence of the chemical reaction network on physical parameters, it is also useful to adopt more realistic models of temperature and density evolution in order to evaluate the temporal and spatial variation of molecular abundances in star-forming cores. Masunaga \& Inutsuka (2000), for example, constructed a one-dimensional (spherical) non-gray radiation-hydrodynamics code to calculate the physical evolution of a dense cloud core to form a protostar. Combining the chemical reaction network model with such radiation-hydrodynamics models, we can investigate temporal and spatial variation of molecular abundances in a star forming core (Aikawa et al. 2008).

In the following, we will present our model results on a collapsing prestellar core, which reproduces the observed chemical fractionation of C-bearing and N-bearing species ( $\$ 2)$. In $\S 3$ we investigate the molecular evolution from a prestellar core to a protostellar core, and compare our model results with the observations of large organic species, carbonchain species and $\mathrm{HCO}_{2}^{+}$in protostellar cores.

\section{Prestellar Cores}

Over the last several years, radio astronomers found chemical fractionation in prestellar cores. While the intensity map of dust continuum and nitrogen-bearing species $\left(\mathrm{N}_{2} \mathrm{H}^{+}\right.$ and $\mathrm{NH}_{3}$ ) are centrally peaked, the map of carbon-bearing species show a central hole. Comparison of these intensity maps revealed that C-bearing species are heavily depleted at the central regions, while N-beating species are not. Figure $2 a$ shows the radial distribution of molecular abundances in L1517B (Tafalla et al. 2002).

In order to investigate the cause of this fractionation, we calculated molecular evolution in prestellar cores by combining the chemical reaction network model with the gravitational contraction of a spherical core. Since the radiation cooling is more efficient than the contraction heating at this evolutionary stage $\left(n_{\mathrm{H}} \leqslant 10^{7} \mathrm{~cm}^{-3}\right)$, we adopt the isothermal collapse model to derive the temporal variation of density in infalling shells, in which the cheical reaction network is solved (Aikawa et al. 2005). Our present model includes two important updates from Aikawa et al. (2005). Firstly, we set the adsorption 
energy of $\mathrm{CO}$ and $\mathrm{N}_{2}$ to be $E_{\text {ads }}(\mathrm{CO})=1180 \mathrm{~K}$ and $E_{\text {ads }}\left(\mathrm{N}_{2}\right)=1060 \mathrm{~K}$. While previous model works often assumed significantly lower adsorption energy of $\mathrm{N}_{2}$ than that of $\mathrm{CO}$, e.g. $E_{\text {ads }}(\mathrm{CO})=1780 \mathrm{~K}$ and $E_{\text {ads }}\left(\mathrm{N}_{2}\right)=750 \mathrm{~K}$, Öberg et al. (2005) found via laboratory experiments that the adsorption energy $E_{\text {ads }}$ of $\mathrm{CO}$ and $\mathrm{N}_{2}$ are similar. Secondly, we assume $\mathrm{N}_{2} \mathrm{H}^{+}$recombination produces $\mathrm{NH}+\mathrm{H}(10 \%)$ and $\mathrm{N}_{2}+\mathrm{H}(90 \%)$, following the recent laboratory experiment (Geppert priv. com.). Previously, the branching ratio was claimed to be $65 \%$ for $\mathrm{NH}+\mathrm{H}$ and $35 \%$ for $\mathrm{N}_{2}+\mathrm{H}$ (Geppert et al. 2004).

Figure $2 \mathrm{~b}$ shows radial distributions of molecular abundances in our model when the central density of the core is similar to that of L1517B $\left(\sim 10^{5} \mathrm{~cm}^{-3}\right)$. The model shows a reasonable agreement with the observation; in the central region, $\mathrm{NH}_{3}$ abundance is slightly enhanced, and $\mathrm{N}_{2} \mathrm{H}^{+}$abundance is almost constant, while $\mathrm{CO}$ is depleted. In our model, $\mathrm{CO}$ is adsorbed onto grains. Since $\mathrm{CO}$ is the main reactant of $\mathrm{N}_{2} \mathrm{H}^{+}$, the rate of $\mathrm{N}_{2} \mathrm{H}^{+}$destruction decreases as $\mathrm{CO}$ depletes. Although $\mathrm{N}_{2}$ can freeze-out onto grains with a similar adsorption energy of $\mathrm{CO}$, slow (i.e. long-lasting) $\mathrm{N}_{2}$ formation also helps to keep the abundance of N-bearing species undepleted (Aikawa et al. 2005, Maret et al. 2006).

\section{Protostellar cores}

\subsection{Physical and chemical evolution}

As the core contracts further, the contraction heating overwhelms the radiation cooling, and core temperature rises. Eventually, the protostar is formed, which further heats the envelope (i.e. protostellar core). In order to investigate the molecular evolution in protostellar cores, we adopt a core model of Masunaga \& Inutsuka (2000). The model starts from a hydrostatic cloud core with the central density of $n_{\mathrm{H}} \sim 6 \times 10^{4} \mathrm{~cm}^{-3}$, and follows the core evolution until $9.3 \times 10^{4}$ yrs after the protostar is born $\left(\equiv t_{\text {final }}\right)$. Although the original paper by Masunaga \& Inutsuka (2000) mainly discusses the formation of a protostar, the model gives the radial distribution of density, temperature and infall velocity in the entire core as a function of time. Figure 3 shows the structure of the core model at assorted evolutionary stages. It should be noted that $\mathrm{CO}$ and large organic species such as $\mathrm{CH}_{3} \mathrm{OH}$ are sublimated to the gas phase at $\sim 20 \mathrm{~K}$ and $\sim 100 \mathrm{~K}$, respectively. At the moment of protostar birth $(t=0)$, for example, CO is sublimated inside $\sim 100$ AU.
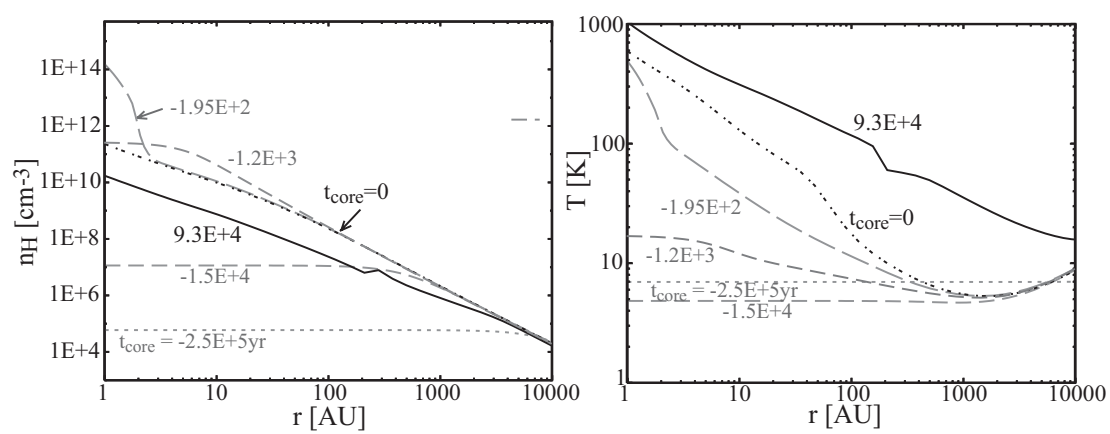

Figure 3. Radial distribution of density and temperature in a star-forming core at assorted evolutionary stages. The protostar is born at $t=0 ; t<0$ (gray lines) corresponds to the prestellar stage, while $t>0$ (black lines) corresponds to the protostellar stage. The core is initially almost isothermal, but the central region start to warm up as the contraction heating overwhelms the radiation cooling $\left(t \sim-1.2 \times 10^{3} \mathrm{yr}\right)$. The first core, i. e. an AU-sized hydrostatic core, appears at $t \sim-$ several $100 \mathrm{yr}$, which then collapses to form a protostar. 

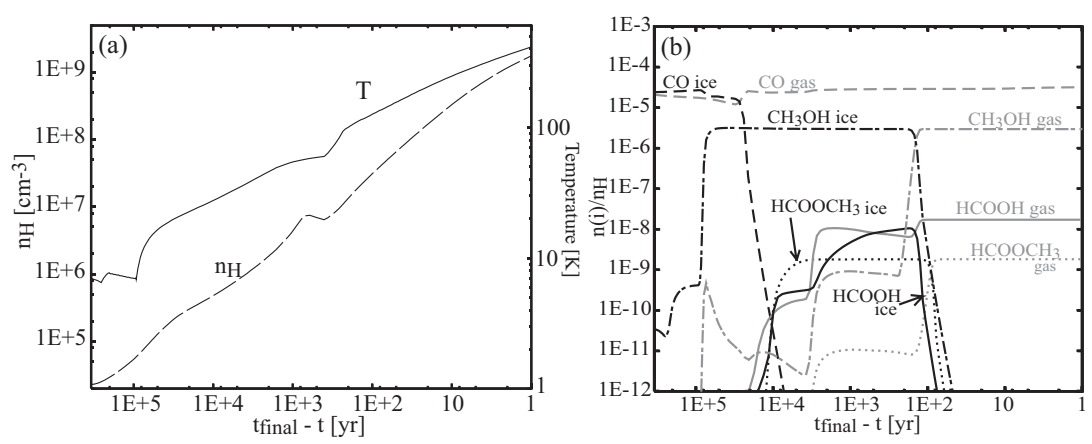

Figure 4. (a) Temporal variation of density and temperature in the infalling shell which reaches $r=2.5 \mathrm{AU}$ at $t_{\mathrm{fina}}$. (b) Evolution of molecular abundances in the shell. Black lines represent the species adsorbed onto grain surfaces (i.e. ice), while gray lines represent gaseous species.

Based on the core model described above, we derive the temporal variation of density and temperature in infalling shells; Figure 4a shows such variation in the shell which reaches $r=2.5 \mathrm{AU}$ at $t_{\text {final }}$. The temporal variation accelerates as the shell migrates towards the inner radius, where the infall velocity, temperature, and density steeply increases inwards. In order to highlight the rapid temporal variation near and at the final stage, the horizontal axis of Figure 4 is set to be the logarithm of $t_{\text {final }}-t$. We can see that once the shell enters the region of $T \geqslant 100 \mathrm{~K}$, it falls onto the central star within $\sim 100$ yrs, which is much shorter than the timescale $\left(\sim 10^{5} \mathrm{yr}\right)$ needed to form the large organic species from sublimated $\mathrm{CH}_{3} \mathrm{OH}$ via gas-phase reactions $(\S 1)$.

Figure $4 b$ shows the molecular evolution in the infalling shell. As initial molecular abundances, we adopt the "low-metal" elemental abundances and assumed that the species are in the form of atoms or atomic ions except for hydrogen, which is in its molecular form. Assuming that the initial cloud core is stable for $1 \times 10^{6} \mathrm{yrs}$, we solved the chemical reaction network with the fixed density and temperature over this period, which sets the initial molecular abundances for the collapse stage shown in Figure 4. We can see that large organic species such as $\mathrm{HCOOCH}_{3}$ are mainly formed on the grain surfaces at $T \sim 20-40 \mathrm{~K}$, and then sublimate to the gas-phase when the shell temperature reaches their sublimation temperatures. One exception is $\mathrm{HCOOH}$, which is formed by the gas-phase reaction of sublimated $\mathrm{H}_{2} \mathrm{CO}$ with $\mathrm{OH}$.

Following the molecular evolution in multiple infalling shells, we obtain spatial distribution of molecules in a protostellar core. Figure 5 shows the radial distribution of molecular abundances at $t_{\text {final }}$. Gaseous organic species are abundant inside about 100 AU, where the temperature exceeds their sublimation temperatures. Formic acid is again an exception; it extends beyond its sublimation radius because of the gas-phase formation. The organic species on grain surfaces are abundant at 100 AU-1,000 AU.

\subsection{Effect of UV radiation: embedded core vs isolated core}

Although we are interested in the central region of protostellar cores, the shells are initially (in the prestellar core stage) at outer radii $r \sim 10^{4} \mathrm{AU}$. In the model described in the previous subsection, we assumed that the model core is embedded in ambient clouds, and set the visual extinction of $A_{\mathrm{v}}=3 \mathrm{mag}$ at the outer boundary of the core. But this assumption is arbitrary; Bok Globules, for example, are not surrounded by ambient clouds and thus can be exposed directly to interstellar UV radiation. In order 
Table 1. Gas-phase molecular abundances in IRAS 16293-2422 and model results.

\begin{tabular}{|c|c|c|c|}
\hline \multirow{2}{*}{ Species } & \multirow{2}{*}{ IRAS $16293-2422$} & \multicolumn{2}{|c|}{ model } \\
\hline & & embedded & isolated $^{a}$ \\
\hline $\mathrm{H}_{2} \mathrm{CO}$ & $1.0(-7)^{b}, 1.1(-7)^{c}$ & $2.8(-6)$ & $1.3(-11)$ \\
\hline $\mathrm{CH}_{3} \mathrm{OH}$ & $1.0(-7)^{d}, 9.4(-8)^{c}$ & $3.0(-6)$ & $8.5(-8)$ \\
\hline $\mathrm{HCOOCH}_{3}$ & $2.5-5.5(-7)^{e}, 2.6-4.3(-9)^{f},>1.2(-8)^{g}$ & $1.8(-9)$ & $3.2(-11)$ \\
\hline $\mathrm{HCOOH}$ & $6.2(-8)^{e}, 2.5(-9)^{g}$ & $1.7(-8)$ & $2.1(-8)$ \\
\hline $\mathrm{CH}_{3} \mathrm{OCH}_{3}$ & $2.4(-7)^{e}, 7.6(-8)^{c}$ & $3.5(-10)$ & $5.8(-12)$ \\
\hline $\mathrm{CH}_{3} \mathrm{CN}$ & $1.0(-8)^{e}, 7.5(-9)^{h}$ & $3.0(-8)$ & $1.3(-8)$ \\
\hline
\end{tabular}

Notes:

${ }^{a}$ Gas-phase abundances at $r=30.6 \mathrm{AU}$ are listed, because the abundances are mostly constant at $r \lesssim 100 \mathrm{AU}$.

${ }^{b}$ Maret et al. (2004), ${ }^{c}$ Chandler et al. (2005), ${ }^{d}$ Maret et al. (2005), ${ }^{e}$ Cazaux et al. $(2003),{ }^{f}$ Kuan et al. $(2004)$,

${ }^{g}$ Remijian \& Hollis (2006), ${ }^{h}$ Schöier et al. (2002).

to investigate the effect of UV radiation on core chemistry, we calculated a model with $A_{\mathrm{v}}=0$ mag at the outer core edge.

We found that the isolated core model evolves to be a protostelolar core with higher abundance of $\mathrm{CO}_{2}$ and lower abundance of large organic molecules compared with the embedded core model (Aikawa et al. 2008). While the shells are at outer radii $\left(\sim 10^{4} \mathrm{AU}\right)$ of the prestellar core, $\mathrm{CO}_{2}$ are efficiently formed by the reactions of

$$
\begin{aligned}
& \mathrm{H}_{2} \mathrm{O}+h \nu \rightarrow \mathrm{H}+\mathrm{OH} \\
& \mathrm{CO}+\mathrm{OH} \rightarrow \mathrm{CO}_{2}+\mathrm{H}
\end{aligned}
$$

on the grain surfaces. In the observation of protostellar cores, molecular abundances are known to vary among objects. Our results indicate that such variation could, at least partially, result from the incident UV radiation at the prestellar stage.

\subsection{Comparison with observation}

Table 1 compares molecular abundances in our embedded and isolated core models and those estimated from the observations of a class 0 object IRAS 16293-2522. It should be noted that the estimated abundances vary significantly depending on the assumptions on temperature and density distribution in the protostellar core. Considering such uncertainties, our models are in reasonable agreement with the observations.

In order to understand the chemical conditions in protostellar cores, it is also important to observe species other than large organic species. Recently, interesting chemical species are detected towards L1527, which is considered to be in the transition phase from class 0 to class I. Firstly, Sakai et al. (2008a) detected carbon chains (e.g., $\mathrm{C}_{4} \mathrm{H}, \mathrm{C}_{4} \mathrm{H}_{2}$, and $\left.\mathrm{C}_{3} \mathrm{H}_{2}\right) ; \mathrm{C}_{4} \mathrm{H}$ emission extends over the 40 " scale, and the line width toward the core center is broader than those toward the surrounding positions, indicating that $\mathrm{C}_{4} \mathrm{H}$ emission

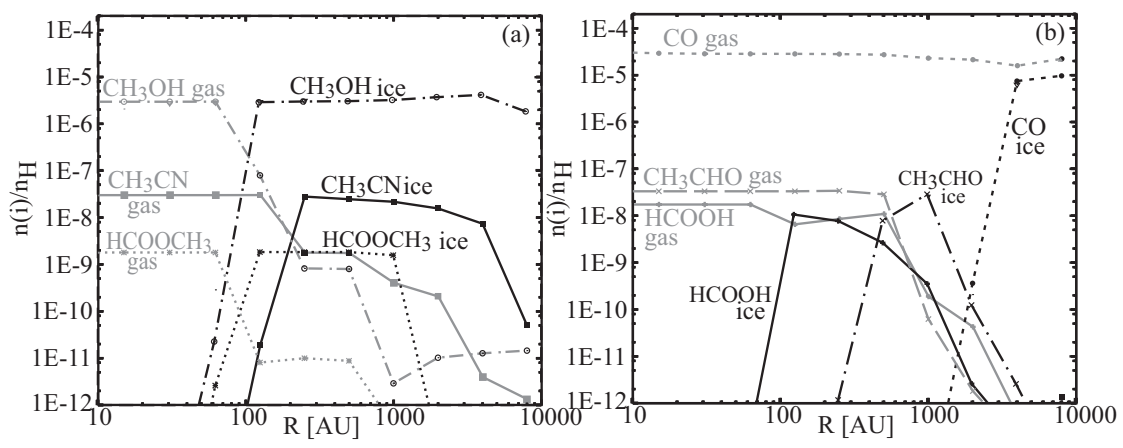

Figure 5. Radial distribution of molecules in our protostellar core model at $t_{\text {final }}$. 


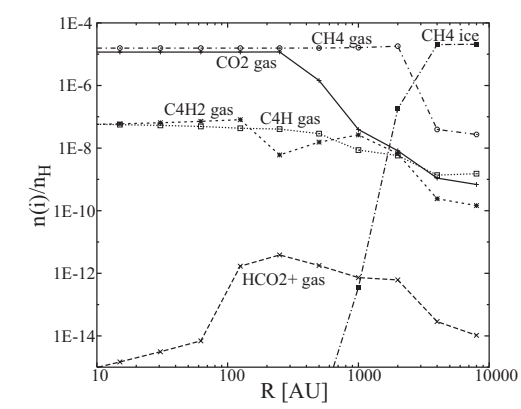

Figure 6. Radial distribution of carbon chains and $\mathrm{HCO}_{2}^{+}$in our protostellar core model at $t_{\text {final }}$.

is continuously distributed from the infalling envelope to the inner part of the core. Existence of carbon-chain species towards L1527 was a surprise, because carbon-chain species are usually associated with the early stages of cloud cores when the dominant form of carbon changes from atomic carbon to CO. Sakai et al. (2008a) proposed that carbon chains could regenerate from sublimated $\mathrm{CH}_{4}$ in the warm central region. Indeed, carbon chains are abundant in our model (Figure 6). Methane is produced in the prestellar core phase and sublimate as the infalling shell temperature rises to $25 \mathrm{~K}$. Then a fraction of sublimated $\mathrm{CH}_{4}$ is transformed to carbon chains via both gas-phase and grain-surface reactions.

Secondly, Sakai et al. (2008b) detected $\mathrm{HCO}_{2}^{+}$towards L1517. While $\mathrm{CO}_{2}$ ice is known to be abundant in protostellar cores (ex. 22\% relative to $\mathrm{H}_{2} \mathrm{O}$ ice towards Elias 29)(Ehrenfreund \& Shutte 2000), the abundance of gaseous $\mathrm{CO}_{2}$ has not been well-constrained. Since $\mathrm{HCO}_{2}^{+}$is formed by the reaction of $\mathrm{H}_{3}^{+}+\mathrm{CO}_{2} \rightarrow \mathrm{HCO}_{2}^{+}+\mathrm{H}_{2}$, it can be a tracer of gaseous $\mathrm{CO}_{2}$. Although the spatial distribution of $\mathrm{HCO}_{2}^{+}$is not well-constrained from the observation, Sakai et al. (2008b) argue, with a help of simple chemical analysis, that the $\mathrm{HCO}_{2}^{+}$seems to extend upto $\sim 2000 \mathrm{AU}$. The column density of $\mathrm{HCO}_{2}^{+}$is estimated to about $7.6 \times 10^{10} \mathrm{~cm}^{-2}$.

In comparison, distribution of $\mathrm{HCO}_{2}^{+}$in our model is shown in Figure 6; it is abundant at radius of $100 \mathrm{AU}<r<2000 \mathrm{AU}$. Column density of $\mathrm{HCO}_{2}^{+}$reaches $10^{10}-10^{11} \mathrm{~cm}^{-2}$ towards the core center (impact parameter $\leqslant 2000 \mathrm{AU}$ ), which is consistent with the observation. In our model, gaseous $\mathrm{CO}_{2}$ and $\mathrm{HCO}_{2}^{+}$increase at the region of $\sim 25 \mathrm{~K}$, where some carbon-chains react with $\mathrm{O}_{2}$ to form $\mathrm{CO}_{2}$ in the gas-phase. $\mathrm{CO}_{2}$ ice is abundantly formed by grain-surface reactions, but they do not desorb efficiently until the shell temperature reaches $\sim 70 \mathrm{~K}$. In the central region of $r \leqslant 100 \mathrm{AU}$, on the other hand, $\mathrm{CO}_{2}$ gas is abundant but $\mathrm{HCO}_{2}^{+}$is not, because $\mathrm{NH}_{3}$ and $\mathrm{H}_{2} \mathrm{O}$, which have larger proton affinity than $\mathrm{CO}_{2}$, are sublimated to the gas phase.

\section{References}

Aikawa, Y., Herbst, E., Roberts, H., \& Caselli, P. 2005, ApJ, 620, 330

Aikawa, Y., Wakelam, V., Herbst, E., \& Garrod, R. T. 2008, ApJ, 674, 984

Chandler, C. J., Brogen, C. L., Shirley, Y. L., \& Loinard, L. 2005, ApJ, 632, 371

Cazaux, E., Tielens, A. G. G. M., Ceccarelli, C., Castets, A., Wakelam, V., Caux, E., Parise, B., \& Teyssier, D. 2003, ApJ (Letter), 593, L51

Ehrenfreund, P. \& Shutte, W. A. 2000, in Astrochemistry: From Molecular Clouds to Planetary Systems, (Chelsea, MI; Sheridan Books; Astronomical Society of the Pacific), p. 135

Garrod, R. T. \& Herbst, E. 2006, AESA, 457, 927

Geppert, W. D., et al. 2004, ApJ, 609, 459 
Geppert, W. D., Thomas, R. D., Ehlerding, A., et al. 2006, Faraday Discuss., 133, 177

Horn, A., Møllendal, H., Sekiguchi, O., et al. 2004, ApJ, 611, 605

Kuan, Y.-J., Juang, H.-C., Charnley, S. B., Hirano, N., Takakuwa, S., Wilner, D. J., Liu, S.-Y., Ohashi, N., Bourke, T. L., Qi, C., \& Zhang, Q. 2004, ApJ (Letter), 616, L27

Maret, S., Bergin, E. A., \& Lada, C. J. 2006, Nature, 442, 425

Maret, S., Ceccarelli, C., Caux, E., Tielens, A. G. G. M., Jørgensen, J. K., van Dishoeck, E. F., Bacmann, A., Castets, A., Lefloch, B., Loinard, L., Parise, B., \& Schöier, F. L. 2004, A\&A, 416,577

Maret, S., Ceccarelli, C., Tielens, A. G. G. M., Caux, E., Lefloch, B., Faure, A., Castet, A., \& Flower, D. R. 2005, $A \& \mathcal{G} A, 442,527$

Masunaga, H. \& Inutsuka, S. 2000, ApJ, 531, 350

Millar, T. J. \& Hatchell J. 1998, Faraday Discuss., 109, 15

Öberg, K., van Broekhuizen, F., Fraser, H. J., Bisschop, S. E., van Dishoeck, E. F., \& Schlemmer, S. 2005, ApJ (Letter), 621, L33

Remijian, A. J. \& Hollis, J. M. 2006, ApJ, 640, 842

Sakai, N., Sakai, T., Aikawa, Y., \& Yamamoto, S. 2008b, ApJ (Letter), 675, L89

Sakai, N., Sakai, T., Hirota, T., \& Yamamoto, S. 2008a, ApJ, 672, 371

Schöier, F. L., Jørgensen, J. K., van Dishoeck, E. F., \& Blake, G. A. 2002, A\& A, 391, 1001

Tafalla, M., Myers, P. C., Caselli, P., Walmsley, C. M., \& Comito, C. 2002, ApJ, 569, 815

\section{Discussion}

BotTINELLI: I was wondering what abundance of ammonia you had in your model and whether you can see difference in the chemistry between ammonia rich and ammonia poor ice.

AIKAWA: In our model the ammonia abundance is not assumed. It is determined by the chemical reaction network, and is about $10 \%$ of $\mathrm{H}_{2} \mathrm{O}$ ice in outer radius.

Cuppen: I have a question about the accretion model, your updated version. You show that ammonia is in agreement with the observations. Can you comment on the CO?

AIKAWA: CO in the gas phase is determined by the efficiency of cosmic ray desorption. This is one of the assumptions in the model. Observationally, we do not have a sensitivity to distinguish between $10^{-7}$ abundance to $10^{-6}$ abundance. Observers can only say that it is lower than $10^{-6}$.

CECCARELLI: I saw that your model prediction of the abundance of large organic molecules is around 100 times lower than we observed.

AIKAWA: In our model we need methanol to produce these molecues. I want to emphasize that there is no assumptions on initial conditions or ice abundance. I just started with a very simple protostellar core and solved detailed but still simple chemical reaction network, to see how far we can go and how much organic species we can make. If you need more, I have to work on the chemical network to make more organics from methanol. Another solution could be axisymetric model, rather than spherical. Then the fluid parcels can stay longer in warm regions (of circumstellar disk) in which large organic molecules are formed. 